\title{
HARDY-ORLICZ SPACES OF CONFORMAL DENSITIES
}

\author{
SITA BENEDICT
}

Abstract. We define and prove characterizations of Hardy-Orlicz spaces of conformal densities.

\section{INTRODUCTION}

In 9 the authors defined a new type of Hardy-Orlicz space by considering the internal path distance from $f(x)$ to $f(0)$ in place of the euclidean distance $|f(x)|$, where $f$ is a conformal mapping of $\mathbb{B}^{2}$. The internal distance between two points $f(x), f(y) \in f\left(\mathbb{B}^{2}\right)$ is formally

$$
d_{I}(f(x), f(y))=\inf _{\gamma} \int_{\gamma}\left|f^{\prime}\right| d s,
$$

where $d s$ denotes integration with respect to arc length and the infimum is taken over all curves in $\mathbb{B}^{2}$ with endpoints $x$ and $y$. Thus $d_{I}$ is a metric on $f\left(\mathbb{B}^{2}\right)$, but it can equivalently be thought of as a metric on $\mathbb{B}^{2}$ associated with the conformal mapping $f$. Since the definition depends on $\left|f^{\prime}\right|$ and not on $f$ we can think of $\left|f^{\prime}\right|$ as a special kind of density on $\mathbb{B}^{2}$ and ask what are the properties of $\left|f^{\prime}\right|$ that are actually needed to develop the theory of intrinsic Hardy-Orlicz spaces. In general, a density on $\mathbb{B}^{n}$ is simply a Borel measurable function $\rho: \mathbb{B}^{n} \rightarrow[0, \infty]$. For a given density $\rho$ the $\rho$-length of a curve $\gamma$ in $\mathbb{B}^{n}$ is

$$
\operatorname{length}_{\rho}(\gamma)=\int_{\gamma} \rho(x) d s
$$

If $\rho$ is continuous and strictly positive we can define the metric $d_{\rho}$ on $\mathbb{B}^{n}$ by setting

$$
d_{\rho}(x, y)=\inf \text { length }_{\rho}(\gamma), x, y \in \mathbb{B}^{n},
$$

where the infimum is taken over all curves $\gamma \subset \mathbb{B}^{n}$ with endpoints $x$ and $y$.

It was shown in 3 that a continuous density $\rho: \mathbb{B}^{n} \rightarrow(0, \infty)$ need only satisfy two simple conditions so that a number of classical results from geometric function theory in the plane generalize to the setting of conformal densities on $\mathbb{B}^{n}$. The first is a Harnack-type inequality (HI), where $\rho$ does not vary much on Whitney-type scales in the ball. We say that $\rho$ satisfies $\operatorname{HI}(A)$ if there exists a constant $A \geq 1$ such that for all $z \in \mathbb{B}^{n}$,

$$
1 / A \leq \frac{\rho(x)}{\rho(y)} \leq A, \text { whenever } x, y \in B_{z}=B(z,(1-|z|) / 2) .
$$

Received by the editors September 23, 2014 and, in revised form, March 19, 2015.

2010 Mathematics Subject Classification. Primary 30C35, 30H10.

Key words and phrases. Hardy spaces, Hardy-Orlicz, conformal densities.

The author was partially supported by the Academy of Finland grants 131477 and 263850. 
The second condition is a Volume Growth condition (VG). To state this we associate with $\rho$ a Borel measure $\mu_{\rho}$ on $\mathbb{B}^{n}$ by setting

$$
\mu_{\rho}(E)=\int_{E} \rho^{n} d x
$$

for each Borel set $E \subseteq \mathbb{B}^{n}$, and we say that $\rho$ satisfies condition $\operatorname{VG}(B)$ if there exists a constant $B>0$ such that

$$
\mu_{\rho}\left(B_{\rho}(x, r)\right) \leq B r^{n}
$$

for all $x \in \mathbb{B}^{n}$ and $r>0$, where $B_{\rho}(x, r)=\left\{y \in \mathbb{B}^{n}: d_{\rho}(x, y)<r\right\}$. A conformal density on $\mathbb{B}^{n}$ is then any continuous density $\rho: \mathbb{B}^{n} \rightarrow(0, \infty)$ satisfying both $\operatorname{HI}(A)$ and $\operatorname{VG}(B)$.

It is simple to show that $\left|f^{\prime}\right|$ is a conformal density with constants $A=e^{12}$ and $B=\pi$ whenever $f$ is a conformal mapping of $\mathbb{B}^{2}$. If $g: \mathbb{B}^{n} \rightarrow \mathbb{R}^{n}$ is quasiconformal, then it can be shown that the averaged derivative of $g$, usually denoted as $a_{g}$, is a conformal density on $\mathbb{B}^{n}$. For details and other examples of conformal densities, including ones that do not arise from a quasiconformal mapping, see 3].

We show in this paper that the same two conditions $\mathrm{HI}(A)$ and $\operatorname{VG}(B)$ are sufficient to develop a Hardy-Orlicz space theory for conformal densities on the unit ball in $\mathbb{R}^{n}$.

Let $\psi:[0, \infty] \rightarrow[0, \infty]$ be a strictly increasing, differentiable function with $\psi(0)=0$, or growth function for short. We say that a conformal density $\rho$ on $\mathbb{B}^{n}$ belongs to the Hardy-Orlicz space $H^{\psi}$ if there is a $\delta>0$ such that

$$
\sup _{0<r<1} \int_{\mathbb{S}^{n-1}} \psi\left(\delta|(r \omega)|_{\rho}\right) d \sigma(\omega)<\infty
$$

where the distance $|(r \omega)|_{\rho}$ between $r \omega$ and 0 is the one induced by $\rho$, and $\sigma$ is the $n$-1-dimensional surface measure on $\mathbb{S}^{n-1}$. If $\psi(t)=t^{p}$ for a given $0<p<\infty$ then we simply denote the corresponding Hardy space with the symbol $H^{p}$. Our first result gives several characterizations of these spaces that hold for all growth functions $\psi$.

Theorem 1.1. Let $\rho$ be a conformal density on $\mathbb{B}^{n}$ and $\psi$ a growth function. Then the following are equivalent:

(1) $\rho \in H^{\psi}$,

(2) $\psi\left(\delta_{1}|\omega|_{\rho}\right) \in L^{1}\left(\mathbb{S}^{n-1}\right)$ for some $\delta_{1}>0$,

(3) $\psi\left(\delta_{2} \rho^{*}(\omega)\right) \in L^{1}\left(\mathbb{S}^{n-1}\right)$ for some $\delta_{2}>0$,

(4) $(1-r)^{n-2} \psi\left(\delta_{3} M(r, \rho)\right) \in L^{1}((0,1))$ for some $\delta_{3}>0$.

The definitions of the non-tangential maximal function $\rho^{*}$ and the maximum modulus $M(r, \rho)$ are given in Section 3. The characterizations in Theorem 1.1 are analogues to results that hold for the classical Hardy spaces of holomorphic functions on the unit disk, which follow from results in [10, 12 and 7 . We recall here the definition of these spaces. A holomorphic mapping $f$ of $\mathbb{B}^{2}$ belongs to the classical Hardy space $\mathcal{H}^{p}, 0<p<\infty$, if

$$
\sup _{0<r<1} \int_{\mathbb{S}^{1}}|f(r \omega)|^{p} d \sigma(\omega)<\infty .
$$

In the classical setting the characterization involving the maximum modulus holds only when restricting to conformal (holomorphic and injective) mappings of $\mathbb{B}^{2}$ 
belonging to $\mathcal{H}^{p}$. In 1 the authors established a theory of Hardy spaces of quasiconformal mappings of $\mathbb{B}^{n}$ and generalized the maximum modulus characterization and other classical Hardy space results to this setting. For more on the classical Hardy spaces, see [5].

Other characterizations that hold for the classical $\mathcal{H}^{p}$ spaces when restricting to the conformal mappings have been established in more recent years. For instance, it was established in both [1] and [2] that if $f$ is a conformal mapping of $\mathbb{B}^{2}$, then

$$
f \in \mathcal{H}^{p} \text { if and only if } \int_{\mathbb{B}^{2}}\left|f^{\prime}(x)\right|^{p}(1-|x|)^{p-1} d x<\infty,
$$

for all $0<p<\infty$. We show, as a corollary to statements proved for more general growth functions, that the corresponding statement for conformal densities is also true; see Theorem 1.2 below. Our result, in combination with a theorem from 9 which says that the internal Hardy spaces and classical Hardy space classes of conformal mappings are the same for all $0<p<\infty$, also implies the result (1.1), and so our work gives an alternative, and shorter, proof to the ones found in [1] and [2]. See Section 4.

Theorem 1.2. Let $\rho$ be a conformal density of $\mathbb{B}^{n}$ and $0<p<\infty$. Then

$$
\rho \in H^{p} \text { if and only if } \int_{\mathbb{B}^{n}} \rho(x)^{p}(1-|x|)^{p-1} d x<\infty .
$$

It is well known that a conformal map $f$ on the unit disk belongs to the classical $\mathcal{H}^{p}$ space for all $p<1 / 2$. As a consequence of Theorem 1.1 every conformal density also belongs to $H^{p}$ for all $p$ in a certain range.

Theorem 1.3. There exists a constant $p_{0}=p_{0}(n, A, B)>0$ so that every conformal density $\rho: \mathbb{B}^{n} \rightarrow(0, \infty)$ satisfying $\mathrm{HI}(A)$ and $\operatorname{VG}(B)$ belongs to $H^{p}$ for all $p<p_{0}$.

We obtain as a corollary by way of the Gehring-Hayman theorem (see Section 2) the following.

Corollary 1.4. There exists a constant $p_{0}=p_{0}(n, A, B)>0$ so that

$$
\int_{\mathbb{S}^{n-1}}\left(\int_{0}^{1} \rho(t \omega) d t\right)^{p} d \sigma<\infty
$$

whenever $0<p<p_{0}$ and $\rho$ is a conformal density satisfying $\mathrm{HI}(A)$ and $\operatorname{VG}(B)$.

This paper is organized as follows. Section 2 covers notation, modulus of curve families and also the Gehring-Hayman Theorem. In Section 3 we prove Theorem 1.1. and in Section 4 we prove our results that require an additional assumption on $\psi$.

\section{Preliminaries}

We set $\mathbb{B}^{n}=\left\{x \in \mathbb{R}^{n}:|x|<1\right\}$ and $\mathbb{S}^{n-1}=\left\{x \in \mathbb{R}^{n}:|x|=1\right\}$, and in general, $B(x, r)$ denotes the open ball in $\mathbb{R}^{n}$ centered at $x$ and with radius $r>0$. For each $x \in \mathbb{B}^{n}$ let

$$
B_{x}=B(x,(1-|x|) / 2)
$$

and

$$
S_{x}=\left\{\frac{x}{|x|}: x \in B_{x}, x \neq 0\right\} \subseteq \mathbb{S}^{n-1},
$$


and for each $\omega \in \mathbb{S}^{n-1}$ let

$$
\Gamma(\omega)=\bigcup\left\{B_{t \omega}: 0 \leq t<1\right\}
$$

be the Stolz cone centered at $\omega$. The surface area of $\mathbb{S}^{n-1}$ will be denoted as $\omega_{n-1}$.

Whenever we write a constant as $C=C(A, B, \ldots)$ we mean that the constant depends only on the values $A, B, \ldots$. In a proof the value of a constant can change from one line to the next without any notational indication or explanation. We will write $A \approx B$ to indicate that there exists a constant $C$ such that

$$
\frac{A}{C} \leq B \leq C A
$$

Let $\rho$ be a conformal density on $\mathbb{B}^{n}$ and $d_{\rho}$ the metric on $\mathbb{B}^{n}$ induced by $\rho$. For each $x \in \mathbb{B}^{n}$ we abbreviate

$$
|x|_{\rho}=d_{\rho}(x, 0) .
$$

The metric extends to the boundary in the sense that

$$
d_{\rho}(\omega, x)=\inf \operatorname{length}_{\rho}(\gamma)
$$

is well defined for each $\omega \in \mathbb{S}^{n-1}$ and $x \in \mathbb{B}^{n}$ by taking the infimum over all curves $\gamma$ in $\mathbb{B}^{n}$ with endpoints $\omega$ and $x$. By [3, Theorem 4.4] $d_{\rho}(\omega, x)<\infty$ for any $x \in \mathbb{B}^{n}$ and almost every $\omega \in \mathbb{S}^{n-1}$. We abbreviate also

$$
|\omega|_{\rho}=d_{\rho}(\omega, 0) \text {. }
$$

The subscript $\rho$ will be used to denote the usual metric notions in the metric space $\left(\mathbb{B}^{n}, d_{\rho}\right)$. For example, given $x \in \mathbb{B}^{n}$ and $r>0$ we set $B_{\rho}(x, r)=\left\{y \in \mathbb{B}^{n}\right.$ : $\left.d_{\rho}(x, y)<r\right\}$.

One of our main tools is the modulus of curve families, defined here. Let $\Gamma$ be a family of locally rectifiable curves in $\mathbb{B}^{n}$. The modulus $\operatorname{Mod} \Gamma \in[0, \infty]$ is defined to be

$$
\operatorname{Mod} \Gamma=\inf _{\varrho} \int_{\mathbb{B}^{n}} \varrho^{n} d x
$$

where the infimum is taken over all densities $\varrho: \mathbb{B}^{n} \rightarrow[0, \infty]$ that satisfy length $_{\varrho}(\gamma) \geq 1$ for every $\gamma \in \Gamma$. We clarify that here and in the following lemma the path integrals are calculated with respect to the euclidean metric. For certain families of curves, the exact value of the modulus is easy to calculate. For instance, if $E$ is a Borel set in $\mathbb{S}^{n-1}$ and $\Gamma$ is the collection of radial segments with one endpoint in $B(0, r), 0<r<1$, and the other endpoint in $E$, then

$$
\operatorname{Mod} \Gamma=\sigma(E)(\log (1 / r))^{1-n} .
$$

See 13 for this result and other properties of the modulus.

We will need the following modulus estimate from [3, Lemma 3.2].

Lemma 2.1. Let $\rho$ be a conformal density on $\mathbb{B}^{n}$ satisfying $\operatorname{VG}(B)$. Then there exists a constant $C(B, n) \geq \omega_{n-1}$ with the following property. Let $E$ be a non-empty subset of $\mathbb{B}^{n}$ and suppose $L \geq \delta>0$. Assume that $\operatorname{diam}_{\rho}(E) \leq \delta$ and that $\Gamma$ is a family of curves in $\mathbb{B}^{n}$ so that $\gamma$ has one endpoint in $E$ and length $\rho(\gamma) \geq L$ for every $\gamma \in \Gamma$. Then

$$
\operatorname{Mod} \Gamma \leq \frac{C}{[\log (1+L / \delta)]^{n-1}} .
$$

Now using simple modulus techniques we obtain the following. 
Lemma 2.2. Let $\rho$ be a conformal density. There exists a constant $C=C(n, A, B)$ such that

$$
\sigma\left(\left\{\omega \in S_{x}: d_{\rho}(w, x)>M \rho(x)(1-|x|)\right\}\right) \leq C \sigma\left(S_{x}\right)(\log M)^{1-n}
$$

for any $x \in \mathbb{B}^{n}$ and $M>1$.

Proof. Let $x \in \mathbb{B}^{n}$ and $E=\left\{\omega \in S_{x}: d_{\rho}(w, x)>M \rho(x)(1-|x|)\right\}$. Suppose first that $|x|<1 / 4$. If $\Gamma_{E}$ is the collection of radial segments with one endpoint in $E$ and the other in $B_{x} \cap S(0,1 / 4)$, then $\operatorname{Mod}\left(\Gamma_{E}\right)=\sigma(E)(\log 4)^{1-n}$. By property $\mathrm{HI}(A)$ and the definition of the set $E$ there is a constant $C=C(n, A)$ such that each curve in $\Gamma_{E}$ has one endpoint in $B_{\rho}(x, C \rho(x)(1-|x|))$ and the other in $\overline{\mathbb{B}^{n}} \backslash$ $B_{\rho}(x, M \rho(x)(1-|x|))$. If $2 \leq C$ and $C^{2}<M$, then

$$
\sigma(E)(\log 4)^{1-n}=\operatorname{Mod}\left(\Gamma_{E}\right) \leq C(\log M)^{1-n} \leq C \omega_{n-1}(\log M)^{1-n}
$$

by Lemma 2.1. If $1<M \leq C^{2}$ then trivially

$$
\sigma(E) \leq \omega_{n-1}\left(\log C^{2}\right)^{n-1}(\log M)^{1-n} .
$$

If $1 / 4 \leq|x|$ and $\Gamma_{E}$ is the collection of radial segments with one endpoint in $E$ and the other endpoint in $B_{x} \cap S(0,|x|)$ then $\operatorname{Mod}\left(\Gamma_{E}\right)=\sigma(E)\left(\log \frac{1}{|x|}\right)^{1-n}$. As before, Lemma 2.1 implies that

$$
\sigma(E)(\log 1 /|x|)^{1-n}=\operatorname{Mod}\left(\Gamma_{E}\right) \leq C(\log M)^{1-n}
$$

whenever $2 \leq C$ and $C^{2}<M$. The other case is again trivial, so noting that $(\log 1 /|x|)^{n-1} \approx \sigma\left(S_{x}\right)$ we are done.

The following version of the Gehring-Hayman theorem is a generalization of a result originally proved by Gehring and Hayman in [6]. This version was proved in [3] using the modulus of curve families as a primary tool. Recall that for all $x \in \mathbb{B}^{n}$ the hyperbolic geodesic connecting 0 and $x$ is the radial segment $[0, x]$.

Gehring-Hayman Theorem. Let $\rho$ be a conformal density on $\mathbb{B}^{n}$. There is a constant $C(A, B, n)$ with the following property. If $\gamma$ is a hyperbolic geodesic in $\mathbb{B}^{n}$ with endpoints in $\overline{\mathbb{B}^{n}}$ and $\tilde{\gamma}$ is any other curve in $\mathbb{B}^{n}$ with the same endpoints, then

$$
\operatorname{length}_{\rho}(\gamma) \leq C \operatorname{length}_{\rho}(\tilde{\gamma}) \text {. }
$$

\section{Proof of Theorem 1.1}

For each conformal density $\rho$ define the non-tangential maximal function $\rho^{*}$ on $\mathbb{S}^{n-1}$ as

$$
\rho^{*}(\omega)=\sup _{x \in \Gamma(\omega)}|x|_{\rho} .
$$

Lemma 3.1. Let $\psi$ be a growth function, $\rho$ a conformal density and $\delta>0$. There exists a constant $C=C(A, B, n)$ such that

$$
\int_{\mathbb{S}^{n-1}} \psi\left(\frac{\delta \rho^{*}(\omega)}{C}\right) d \sigma \leq \int_{\mathbb{S}^{n-1}} \psi\left(\delta|\omega|_{\rho}\right) d \sigma .
$$

Proof. Let $\omega \in \mathbb{S}^{n-1}$ and $x \in \Gamma(\omega)$. Then $x \in B_{t \omega}$ for some $0<t<1$. The Gehring-Hayman theorem and $\mathrm{HI}(A)$ imply that

$$
|x|_{\rho} \leq|t \omega|_{\rho}+d_{\rho}(x, t \omega) \leq C \text { length }_{\rho}([0, \omega)) \leq C|\omega|_{\rho},
$$

from which the result easily follows. 
A measure $\mu$ on $\mathbb{B}^{n}$ is called a Carleson measure if there exists a constant $C(\mu)>$ 0 such that

$$
\mu\left(\mathbb{B}^{n} \cap B(\omega, r)\right) \leq C(\mu) r^{n-1}
$$

for all $\omega \in \mathbb{S}^{n-1}$ and all $r>0$. We denote the infimum of all such constants $C(\mu)$ by $\alpha_{\mu}$.

Lemma 3.2. Let $\rho$ be a conformal density, $\psi$ a growth function, $\delta>0$ and $\mu$ a Carleson measure on $\mathbb{B}^{n}$. There are constants $C_{1}=C_{1}(A, B, n)$ and $C_{2}=$ $C_{2}\left(n, \alpha_{\mu}\right)$ such that

$$
\int_{\mathbb{B}^{n}} \psi\left(\frac{\delta|x|_{\rho}}{C_{1}}\right) d \mu \leq C_{2} \int_{\mathbb{S}^{n-1}} \psi\left(\delta|\omega|_{\rho}\right) d \sigma
$$

Proof. Let $\epsilon>0$ and set $E(\lambda)=\left\{x \in \mathbb{B}^{n}: \epsilon|x|_{\rho}>\lambda\right\}$ and $U(\lambda)=\left\{\omega \in \mathbb{S}^{n-1}\right.$ : $\left.\epsilon \rho^{*}(\omega)>\lambda\right\}$ for each $\lambda>0$. We can use the generalized form of the Whitney decomposition [4, Theorem III.1.3] to write the open set $U(\lambda)$ as

$$
U(\lambda)=\bigcup_{k=1}^{\infty} S_{x_{k}},
$$

where the points $x_{k} \in \mathbb{B}^{n}$ are chosen so that each $\omega \in U(\lambda)$ belongs to no more than $N(n)$ caps $S_{x_{k}}$ and also so that $\left(1-\left|x_{k}\right|\right) / C \leq d\left(S_{x_{k}}, \partial U(\lambda)\right) \leq C\left(1-\left|x_{k}\right|\right)$. The constant is a universal constant (even independent of $n$ ) and the distance is the spherical distance on $\mathbb{S}^{n-1}$. It follows that $E(\lambda) \subset \bigcup_{k=1}^{\infty} B\left(x_{k} /\left|x_{k}\right|, C\left(1-\left|x_{k}\right|\right)\right)$ for some absolute constant $C$ (again even independent of $n$ ). Indeed, if $x \in E(\lambda)$, then $\frac{x}{|x|} \in S_{x_{k}}$ for some $k$, and so

$$
d\left(x, x_{k} /\left|x_{k}\right|\right) \leq d(x, x /|x|)+d\left(x /|x|, x_{k} /\left|x_{k}\right|\right) \leq C\left(1-\left|x_{k}\right|\right),
$$

by the properties of the Whitney decomposition and the definition of the caps $S_{x_{k}}$. Thus,

$$
\begin{aligned}
\mu(E(\lambda)) & \leq \sum_{k=1}^{\infty} \mu\left(B\left(x_{k} /\left|x_{k}\right|, C\left(1-\left|x_{k}\right|\right)\right) \cap \mathbb{B}^{n}\right) \\
& \leq C\left(n, \alpha_{\mu}\right) \sum_{k=1}^{\infty}\left(1-\left|x_{k}\right|\right)^{n-1} \\
& \leq C\left(n, \alpha_{\mu}\right) \sum_{k=1}^{\infty} \sigma\left(S_{x_{k}}\right) \leq C\left(n, \alpha_{\mu}\right) \sigma(U(\lambda))
\end{aligned}
$$

Then,

$$
\begin{aligned}
\int_{\mathbb{B}^{n}} \psi\left(\epsilon|(x)|_{\rho}\right) d \mu & =\int_{0}^{\infty} \psi^{\prime}(\lambda) \mu(E(\lambda)) d \lambda \\
& \leq C\left(n, \alpha_{\mu}\right) \int_{0}^{\infty} \psi^{\prime}(\lambda) \sigma(U(\lambda)) d \lambda \\
& =C\left(n, \alpha_{\mu}\right) \int_{\mathbb{S}^{n-1}} \psi\left(\epsilon \rho^{*}(\omega)\right) d \sigma .
\end{aligned}
$$

By applying Lemma 3.1 with an appropriate choice of $\epsilon$ we are done. 
With each conformal density $\rho$ we associate the maximum modulus function

$$
M(r, \rho)=\sup _{|x| \leq r}|x|_{\rho}
$$

defined for $r \in[0,1)$. We define the function over the closed ball rather than the sphere of radius $r$ so that the function is increasing. By the Gehring-Hayman theorem, there is a constant $C(A, B, n)$ such that

$$
\sup _{|x|=r}|x|_{\rho} \leq M(r, \rho) \leq C \sup _{|x|=r}|x|_{\rho} .
$$

Proof of Theorem 1.1. Lemma 3.1 shows that (2) implies (3). By definition (3) implies both (2) and (1). If $C$ is the constant from the Gehring-Hayman theorem, then Fatou's lemma and the Gehring-Hayman theorem imply that

$$
\begin{aligned}
\int_{\mathbb{S}^{n-1}} \psi\left(\frac{\delta|\omega|_{\rho}}{C}\right) d \sigma & \leq \int_{\mathbb{S}^{n-1}} \psi\left(\frac{\delta \operatorname{length}_{\rho}([0, \omega))}{C}\right) d \sigma \\
& =\int_{\mathbb{S}^{n-1}} \liminf _{r \rightarrow 1} \psi\left(\frac{\delta \operatorname{length}_{\rho}([0, r \omega])}{C}\right) d \sigma \\
& \leq \liminf _{r \rightarrow 1} \int_{\mathbb{S}^{n-1}} \psi\left(\frac{\delta \operatorname{length}_{\rho}([0, r \omega])}{C}\right) d \sigma \\
& \leq \sup _{0<r<1} \int_{\mathbb{S}^{n-1}} \psi\left(\delta|r \omega|_{\rho}\right) d \sigma,
\end{aligned}
$$

which shows that (1) implies (2). Thus (1), (2), and (3) are equivalent, and we now proceed to show the equivalence of (2) and (4).

First assume (4). We will show that there is a constant $C>0$ such that

$$
\int_{\mathbb{S}^{n-1}} \psi\left(\frac{\delta}{3}|\omega|_{\rho}\right) d \sigma \leq C \int_{0}^{1}(1-r)^{n-2} \psi(\delta M(r, \rho)) d r .
$$

We start by rewriting the integral on the left as

$$
\int_{\mathbb{S}^{n-1}} \psi\left(\frac{\delta}{3}|\omega|_{\rho}\right) d \sigma=\int_{0}^{\infty} \psi^{\prime}(\lambda) \sigma\left(\left\{\omega \in \mathbb{S}^{n-1}: \frac{\delta}{3}|\omega|_{\rho}>\lambda\right\}\right) d \lambda .
$$

Let $E=\left\{\omega \in \mathbb{S}^{n-1}: \frac{\delta}{3}|\omega|_{\rho}>\lambda\right\}$ for a fixed $\lambda$. We will obtain an upper bound on $\sigma(E)$ using modulus of curve families. Indeed, assuming $E$ is non-empty, there exists a unique $r=r(\lambda)$ such that

$$
r_{\lambda}=\sup \{r \in[0,1): \delta M(r, \rho)=\lambda\},
$$

by the continuity of $\rho$. Denote by $\Gamma_{E}$ the path family consisting of the radial segments connecting $B\left(0, r_{\lambda}\right)$ to $E$. Then,

$$
\operatorname{Mod}\left(\Gamma_{E}\right)=\frac{\sigma(E)}{\left(\log \left(1 / r_{\lambda}\right)\right)^{n-1}} \geq \frac{\sigma(E)}{2\left(1-r_{\lambda}\right)^{n-1}}
$$

when $1 / 2<r_{\lambda}<1$. Since each curve in $\Gamma_{E}$ has one endpoint belonging to $\overline{B_{\rho}(0, \lambda / \delta)}$ and one endpoint in $\overline{\mathbb{B}^{n}} \backslash B_{\rho}(0,3 \lambda / \delta)$, we can apply Lemma 2.1 to obtain a constant $C=C(B, n)$ such that

$$
\sigma(E) \leq C\left(1-r_{\lambda}\right)^{n-1}
$$

whenever $1 / 2<r_{\lambda}<1$ and $r_{\lambda}$ is defined as above. If $\nu$ is the measure on $[0,1]$ defined by $d \nu=(1-t)^{n-2} d t \mathrm{~m}$, then

$$
\nu(\{t \in[0,1]: M(t, \rho)>\lambda / \delta\})=\left(1-r_{\lambda}\right)^{n-1} /(n-1) .
$$


This estimate and Fubini's theorem applied to the right-hand side of (3.2) give

$$
\begin{aligned}
\int_{\mathbb{S}^{n-1}} & \psi\left(\frac{\delta}{3}|\omega|_{\rho}\right) d \sigma \\
& \leq \omega_{n-1} \psi(\delta M(1 / 2, \rho))+C \int_{\delta M(1 / 2, \rho)}^{\infty} \psi^{\prime}(\lambda) \nu(\{t \in[0,1]: M(t, \rho)>\lambda / \delta\}) d \lambda \\
& \leq \omega_{n-1} \psi(\delta M(1 / 2, \rho))+C \int_{0}^{\infty} \psi^{\prime}(\lambda) \int_{\{t \in[0,1]: M(t, \rho)>\lambda / \delta\}}(1-t)^{n-2} d t d \lambda \\
& \leq C \int_{0}^{1}(1-t)^{n-2} \psi(\delta M(t, \rho)) d t
\end{aligned}
$$

which is what we needed to show.

Conversely, assume (2) holds for some $\delta>0$, and choose points $x_{k} \in \mathbb{B}^{n}$ such that $\left|x_{k}\right|=r_{k}=1-2^{-k}$ and $\left|x_{k}\right|_{\rho}=\sup _{|x|=r_{k}}|x|_{\rho}, k=1,2, \ldots$ Given any $\epsilon>0$ we have

$$
\begin{aligned}
\int_{0}^{1}(1-r)^{n-2} \psi(\epsilon M(r, \rho)) d r & \leq 2^{n} \sum_{k=1}^{\infty}\left(2^{-k}\right)^{n-1} \psi\left(\epsilon M\left(r_{k}, \rho\right)\right) \\
& \leq 2^{n} \sum_{k=1}^{\infty}\left(2^{-k}\right)^{n-1} \psi\left(C \epsilon\left|x_{k}\right|_{\rho}\right) \\
& =2^{n} \int_{\mathbb{B}^{n}} \psi\left(C \epsilon|x|_{\rho}\right) d \mu,
\end{aligned}
$$

where $d \mu(x)=\sum_{k=1}^{\infty}(1-|x|)^{n-1} \delta_{x_{k}}$ and $C$ is the constant from (3.1). The measure $\mu$ is a Carleson measure, and so by Lemma 3.2 there are universal constants $C_{1}$ and $C_{2}$ such that

$$
\int_{\mathbb{B}^{n}} \psi\left(C \epsilon|x|_{\rho}\right) d \mu \leq C_{1} \int_{\mathbb{S}^{n-1}} \psi\left(C_{2} \epsilon|\omega|_{\rho}\right) d \sigma .
$$

The proof is finished by letting $\epsilon=\delta / C_{2}$.

\section{Characterizations Under additional COnditions ON $\psi$}

A growth function is doubling if there exists a constant $C$ such that $\psi(2 t) \leq$ $C \psi(t)$ for all $t \in[0, \infty]$. The infimum of all such constants is called the doubling constant of $\psi$ and is denoted by $C_{\psi}$.

Lemma 4.1. Let $\psi$ be a doubling growth function and $\rho$ a conformal density. If

$$
\int_{\mathbb{B}^{n}} \psi(\rho(x)(1-|x|)) \frac{d x}{1-|x|}<\infty
$$

then $\psi\left(|\omega|_{\rho}\right) \in L^{1}\left(\mathbb{S}^{n-1}\right)$.

Proof. Our first step is to show that the given assumptions imply that

$$
\int_{\mathbb{S}^{n-1}} \psi(v(\omega)) d \sigma<\infty
$$


where $v(\omega)=\sup _{x \in \Gamma(\omega)}(\rho(x)(1-|x|))$. To that end, fix $\omega \in \mathbb{S}^{n-1}$ and let $x \in \Gamma(\omega)$. Then there is a constant $C=C\left(A, n, C_{\psi}\right)$ such that

$$
\begin{aligned}
\psi(\rho(x)(1-|x|)) & \leq \frac{C}{(1-|x|)^{n}} \int_{B_{x}} \psi(\rho(y)(1-|y|)) d y \\
& \leq C \int_{\Gamma(\omega)} \frac{\psi(\rho(y)(1-|y|))}{(1-|y|)^{n}} d y .
\end{aligned}
$$

Thus,

$$
\int_{\mathbb{S}^{n-1}} \psi(v(\omega)) d \sigma(\omega) \leq C \int_{\mathbb{S}^{n-1}} \int_{\Gamma(\omega)} \frac{\psi(\rho(y)(1-|y|))}{(1-|y|)^{n}} d y d \sigma(\omega),
$$

and so it is enough to show that the integral on the right is finite. If $u(y)=$ $\frac{\psi(\rho(y)(1-|y|))}{1-|y|}$, then by the assumption $u$ is integrable on $\mathbb{B}^{n}$ and Fubini's Theorem gives

$$
\begin{aligned}
\int_{\mathbb{S}^{n-1}} \int_{\Gamma(\omega)} u(y)(1-|y|)^{1-n} d y d \sigma(\omega) & =\int_{\mathbb{B}^{n}} u(y)(1-|y|)^{1-n} \int_{\mathbb{S}^{n-1}} \chi_{\Gamma(\omega)}(y) d \sigma(\omega) d y \\
& \approx \int_{\mathbb{B}^{n}} u(y) d y<\infty
\end{aligned}
$$

which completes the first step.

We now use (4.1) to show that $\psi\left(|\omega|_{\rho}\right) \in L^{1}\left(\mathbb{S}^{n-1}\right)$. Let $U(\lambda)=\left\{\omega \in \mathbb{S}^{n-1}\right.$ : $\left.\rho^{*}(\omega)>\lambda\right\}$ for each $\lambda>0$. Since $U(\lambda)$ is an open set we can use the generalized form of the Whitney decomposition to express $U(\lambda)$ as a union of caps $S_{x_{j}}$,

$$
U(\lambda)=\bigcup S_{x_{j}}
$$

where the caps have uniformly bounded overlap and

$$
\left(1-\left|x_{j}\right|\right) / C \leq d\left(S_{x_{j}}, \partial U(\lambda)\right) \leq C\left(1-\left|x_{j}\right|\right) .
$$

If $\omega \in S_{x_{j}}$ and $v(\omega) \leq \gamma$, then by (4.2) and property $\mathrm{HI}(A)$ there exists $\omega^{\prime} \in$ $\mathbb{S}^{n-1} \backslash U(\lambda)$ and a corresponding $x_{j}^{\prime} \in \Gamma\left(\omega^{\prime}\right)$ such that

$$
\left|x_{j}\right|_{\rho} \leq d_{\rho}\left(x_{j}, x_{j}^{\prime}\right)+\left|x_{j}^{\prime}\right|_{\rho} \leq C \gamma+\lambda .
$$

Now let $M>1$ and $\gamma=\frac{\lambda}{(M+1) C}$ and suppose $\omega \in S_{x_{j}}$ with $v(\omega) \leq \gamma$ and $|\omega|_{\rho}>2 \lambda$. Then, by what we showed above and the definition of $v(\omega)$,

$$
d_{\rho}\left(\omega, x_{j}\right) \geq|\omega|_{\rho}-\left|x_{j}\right|_{\rho}>\lambda-C \gamma=M C \gamma \geq M \rho\left(x_{j}\right)\left(1-\left|x_{j}\right|\right)
$$

and, therefore,

$$
\begin{aligned}
\sigma(\{\omega & \left.\left.\in S_{x_{j}}:|\omega|_{\rho}>2 \lambda \text { and } v(\omega) \leq \gamma\right\}\right) \\
& \leq \sigma\left(\left\{\omega \in S_{x_{j}}: d_{\rho}\left(\omega, x_{j}\right)>M \rho\left(x_{j}\right)\left(1-\left|x_{j}\right|\right)\right\}\right) \\
& \leq C \sigma\left(S_{x_{j}}\right)(\log M)^{1-n}
\end{aligned}
$$


by Lemma 2.2. If $|\omega|_{\rho}>2 \lambda$, then $\omega \in U(\lambda)$, and so by the above we have

$$
\begin{aligned}
\sigma(\{\omega & \left.\in \mathbb{S}^{n-1}:|\omega|_{\rho}>2 \lambda\right\} \\
& \leq \sigma\left(\left\{\omega \in U(\lambda):|\omega|_{\rho}>2 \lambda \text { and } v(\omega) \leq \gamma\right\}\right)+\sigma\left(\left\{\omega \in \mathbb{S}^{n-1}: v(\omega)>\gamma\right\}\right) \\
& \leq C \sum_{j} \sigma\left(S_{x_{j}}\right)(\log M)^{1-n}+\sigma\left(\left\{\omega \in \mathbb{S}^{n-1}: v(\omega)>\gamma\right\}\right) \\
& \leq C \sigma(U(\lambda))(\log M)^{1-n}+\sigma\left(\left\{\omega \in \mathbb{S}^{n-1}: v(\omega)>\gamma\right\}\right) .
\end{aligned}
$$

Thus,

$$
\begin{aligned}
\int_{\mathbb{S}^{n-1}} & \psi\left(\frac{1}{2}|\omega|_{\rho}\right) d \sigma=\int_{0}^{\infty} \psi^{\prime}(\lambda) \sigma\left(\left\{\omega \in \mathbb{S}^{n-1}:|\omega|_{\rho}>2 \lambda\right\}\right) d \lambda \\
\leq & \int_{0}^{\infty} \psi^{\prime}(\lambda)\left(C \sigma(U(\lambda))(\log M)^{1-n}\right. \\
& \left.\quad+\sigma\left(\left\{\omega \in \mathbb{S}^{n-1}: v(\omega)>\frac{\lambda}{(M+1) C}\right\}\right)\right) d \lambda \\
= & C(\log M)^{1-n} \int_{\mathbb{S}^{n-1}} \psi\left(\rho^{*}(\omega)\right) d \sigma+\int_{\mathbb{S}^{n-1}} \psi((M+1) C v(\omega)) d \sigma \\
\leq & C(n, A)(\log M)^{1-n} \int_{\mathbb{S}^{n-1}} \psi\left(\rho^{*}(\omega)\right) d \sigma+C\left(M, n, A, C_{\psi}\right) \int_{\mathbb{S}^{n-1}} \psi(v(\omega)) d \sigma
\end{aligned}
$$

where $C_{\psi}$ is the doubling constant of $\psi$. We would like to use Lemma 3.1 to bring the integral involving $\rho^{*}$ to the left side of the inequality, but since both integrals could be infinite we first apply Lemma 3.1 to the above for the conformal densities $\rho_{t}(x)=\rho(t x), 0<t<1$. By choosing $M$ large enough and taking the limit as $t \rightarrow 1$ we obtain

$$
\int_{\mathbb{S}^{n-1}} \psi\left(|\omega|_{\rho}\right) d \sigma \leq C\left(M, n, A, C_{\psi}\right) \int_{\mathbb{S}^{n-1}} \psi(v(\omega)) d \sigma
$$

which completes the proof.

A full converse to Lemma 4.1 is not possible, as the following example shows. Let $p(x) \equiv 1$ on $\mathbb{B}^{n}$. Then $\rho$ is clearly a conformal density and also $\psi\left(|\omega|_{\rho}\right) \in L^{1}\left(\mathbb{S}^{n-1}\right)$ for every growth function $\psi$. If

$$
\psi(t)= \begin{cases}\frac{1}{\log \frac{1}{t}}, & t<1 / 2 \\ \frac{2 t}{\log 2}, & t \geq 1 / 2\end{cases}
$$

then $\psi$ is a growth function that is doubling, while

$$
\int_{\mathbb{B}^{n}} \psi(\rho(x)(1-|x|)) \frac{d x}{1-|x|}=C+C \int_{1 / 2}^{1} \frac{1}{(1-r) \log \frac{1}{1-r}} d r
$$

is infinite. Assuming superadditivity or concavity plus an additional growth restriction on $\psi(t)$ near $t=0$ we obtain converses in the following forms.

Lemma 4.2. Let $\psi$ be a growth function such that $\psi\left(t_{1}\right)+\psi\left(t_{2}\right) \leq \psi\left(t_{1}+t_{2}\right)$ for all $t_{1}, t_{2} \in[0, \infty]$. If $\rho \in H^{\psi}$, then there is $\delta>0$ such that

$$
\int_{\mathbb{B}^{n}} \psi(\delta \rho(x)(1-|x|)) \frac{d x}{1-|x|}<\infty .
$$


Proof. Let $\delta>0$. By switching to polar coordinates, applying property $\mathrm{HI}(A)$, the superadditivity of $\psi$ and the Gehring-Hayman theorem we have that

$$
\begin{aligned}
\int_{\mathbb{B}^{n}} \psi(\delta \rho(x)(1-|x|)) \frac{d x}{1-|x|} & \leq \int_{\mathbb{S}^{n-1}} \int_{0}^{1} \frac{\psi(\delta \rho(t \omega)(1-t))}{1-t} d t d \sigma \\
& \leq \int_{\mathbb{S}^{n-1}} C_{1} \sum_{j} \psi\left(\delta C_{2} \rho\left(t_{j} \omega\right)\left(1-t_{j}\right)\right) d \sigma \\
& \leq \int_{\mathbb{S}^{n-1}} C_{1} \psi\left(\sum_{j} \delta C_{2} \rho\left(t_{j} \omega\right)\left(1-t_{j}\right)\right) \\
& \leq \int_{\mathbb{S}^{n-1}} C_{1} \psi\left(\delta C_{3} \int_{0}^{1} \rho(t \omega) d t\right) d \sigma \\
& \leq \int_{\mathbb{S}^{n-1}} C_{1} \psi\left(\delta C_{4}|\omega|_{\rho}\right) d \sigma .
\end{aligned}
$$

The last integral is finite for an appropriately chosen $\delta$ by Theorem 1.1 .

Note that the growth function from (4.3) does not satisfy the multiplicative assumption in the next lemma.

Lemma 4.3. Let $\psi$ be a growth function that is concave and for which there exists $C>0$ such that $\psi(a b) \leq b \psi(C a)$ whenever $a \geq 0$ and $0<b<1$. Then if $\rho \in H^{\psi}$, then

$$
\int_{\mathbb{B}^{n}} \psi(\rho(x)(1-|x|)) \frac{d x}{1-|x|}<\infty .
$$

Proof. By Jensen's inequality and the multiplicative property of $\psi$ to get that

$$
\begin{aligned}
\psi^{-1}\left(\int_{0}^{1} \frac{\psi(\rho(t \omega)(1-t))}{1-t} d t\right) & =\lim _{r \rightarrow 1} \psi^{-1}\left(\int_{0}^{r} \frac{\psi(\rho(t \omega)(1-t))}{1-t} d t\right) \\
& \leq \int_{0}^{1} C \rho(t \omega) d t .
\end{aligned}
$$

Then by the Gehring-Hayman theorem

$$
\begin{aligned}
\int_{\mathbb{B}^{n}} \psi(\rho(x)(1-|x|)) \frac{d x}{1-|x|} & \leq \int_{\mathbb{S}^{n-1}} \int_{0}^{1} \frac{\psi(\rho(t \omega)(1-t))}{1-t} d t d \sigma \\
& \leq \int_{\mathbb{S}^{n-1}} \psi\left(C \int_{0}^{1} \rho(t \omega) d t\right) d \sigma \\
& \leq \int_{\mathbb{S}^{n-1}} \psi\left(C|\omega|_{\rho}\right) d \sigma,
\end{aligned}
$$

which is finite by Theorem 1.1

Proof of Theorem 1.2. This follows clearly from Lemmas 4.1, 4.2 and 4.3.

In [9] the authors proved that if $f: \mathbb{B}^{2} \rightarrow \mathbb{C}$ is conformal, then $f$ belongs to the classical Hardy space $\mathcal{H}^{p}$ if and only if $\left|f^{\prime}\right| \in H^{p}$ for all $0<p<\infty$. The result from the classical setting stated in (1.1) then follows as a corollary to Theorem 1.2 . The theorem from [9] and the proof of Theorem 1.2 relied primarily on modulus of curve famlies and the use of properties of conformal densities, and so this new proof of (1.1) is shorter and less technical than those in [1] and 22. The earlier 
proofs relied on, for example, the use of Carleson measures in the case of [1] and on several older theorems including that from Pommerenke [11, Hayman [8] and two Hardy-Littlewood inequalities in the case of [2]. We note that especially in one direction, the proofs in the setting of conformal densities are very straightforward.

Proof of Theorem 1.3. Let $\rho$ be a conformal density on $\mathbb{B}^{n}$. By [3, Theorem 5.1] there exist constants $\beta(B, n)>1, C_{1}(A, B, n), C_{2}(A, B, n)$ so that

$$
C_{1}(1-|x|)^{\beta-1} \leq \frac{\rho(x)}{\rho(0)} \leq C_{2} \frac{1}{(1-|x|)^{\beta-1}}
$$

for every $x \in \mathbb{B}^{n}$. Since by the Gehring-Hayman theorem

$$
M(r, \rho) \approx \sup _{\omega \in \mathbb{S}^{n-1}} \int_{0}^{r} \rho(t \omega) d t
$$

for all $0<r<1$, there exists some constant $C>0$ such that

$$
M(r, \rho) \leq C(1-r)^{-\beta} .
$$

Thus,

$$
\int_{0}^{1}(1-r)^{n-2} M(r, \rho)^{p} d r
$$

is finite for any $p<\frac{n-1}{\beta}$ and $\rho \in H^{p}$ for all such $p$ by Theorem 1.1

\section{ACKNOWLEDGEMENT}

This paper forms a part of the thesis of the author, written under the supervision of Pekka Koskela.

\section{REFERENCES}

[1] K. Astala and P. Koskela, $H^{p}$-theory for quasiconformal mappings, Pure Appl. Math. Q. 7 (2011), no. 1, 19-50, DOI 10.4310/PAMQ.2011.v7.n1.a3. MR2900163

[2] A. Baernstein II, D. Girela, and J. Á. Peláez, Univalent functions, Hardy spaces and spaces of Dirichlet type, Illinois J. Math. 48 (2004), no. 3, 837-859. MR2114254 (2005i:30085)

[3] M. Bonk, P. Koskela, and S. Rohde, Conformal metrics on the unit ball in Euclidean space, Proc. London Math. Soc. (3) 77 (1998), no. 3, 635-664, DOI 10.1112/S0024611598000586. MR.1643421 (99f:30033)

[4] R. R. Coifman and G. Weiss, Analyse harmonique non-commutative sur certains espaces homogènes (French), Lecture Notes in Mathematics, Vol. 242, Springer-Verlag, Berlin-New York, 1971. Étude de certaines intégrales singulières. MR.0499948 (58 \#17690)

[5] P. L. Duren, Theory of $H^{p}$ Spaces, Pure and Applied Mathematics, Vol. 38, Academic Press, New York-London, 1970. MR0268655 (42 \#3552)

[6] F. W. Gehring and W. K. Hayman, An inequality in the theory of conformal mapping, J. Math. Pures Appl. (9) 41 (1962), 353-361. MR0148884 (26 \#6381)

[7] G. H. Hardy and J. E. Littlewood, Some properties of fractional integrals. II, Math. Z. 34 (1932), no. 1, 403-439, DOI 10.1007/BF01180596. MR1545260

[8] W. K. Hayman, Multivalent functions, Cambridge Tracts in Mathematics and Mathematical Physics, No. 48, Cambridge University Press, Cambridge, 1958. MR0108586 (21 \#7302)

[9] P. Koskela and S. Benedict, Intrinsic Hardy-Orlicz spaces of conformal mappings, Bull. Lond. Math. Soc. 47 (2015), no. 1, 75-84, DOI 10.1112/blms/bdu097. MR.3312966

[10] F. Nevanlinna and R. Nevanlinna, Über die eigenschaften analytischer Funktionen in der Umgebung einer singulären Stelle oder Linie, Acta Soc. Sci. Fenn. 50 (1922), no. 5.

[11] Ch. Pommerenke, Über die Mittelwerte und Koeffizienten multivalenter Funktionen, Math. Annalen 145 (1962), 185-296. 
[12] H. Prawitz, Über Mittelwerte analytischer Funktionen, Ark. Mat. Astr. Fys. 20A (1927), $1-12$.

[13] J. Väisälä, Lectures on n-dimensional quasiconformal mappings, Lecture Notes in Mathematics, Vol. 229, Springer-Verlag, Berlin-New York, 1971. MR0454009 (56 \#12260)

Department of Mathematics and Statistics, University of Jyväskylä, P.O. Box 35 (MAD), FI-40014, Finland

E-mail address: sita.c.benedict@jyu.fi 